\title{
Erratum: Business Strategies and Competitiveness in Times of Crisis
}

(C) The Editor(s) (if applicable) and The Author(s) 2016

L. Gavinelli, Business Strategies and Competitiveness in Times of Crisis DOI 10.1057/978-1-137-57810-5

The affiliation for the author Dr Laura Gavinelli has been updated as below:

Laura Gavinelli, Di.SEA.DE, Dipartimento di Scienze Economico-Aziendali e Diritto per l'Economia, Università degli Studi di Milano-Bicocca, Milan, Italy

Also, figures 4.1, 4.2, 4.3 and 4.28 in chapter 4 have been replaced with revised ones.

The updated original online version for this chapter can be found at DOI 10.1057/978-1-137-57810-5_6

(C) The Editor(s) (if applicable) and The Author(s) 2016

L. Gavinelli, Business Strategies and Competitiveness in Times of Crisis, DOI 10.1057/978-1-137-57810-5_6 


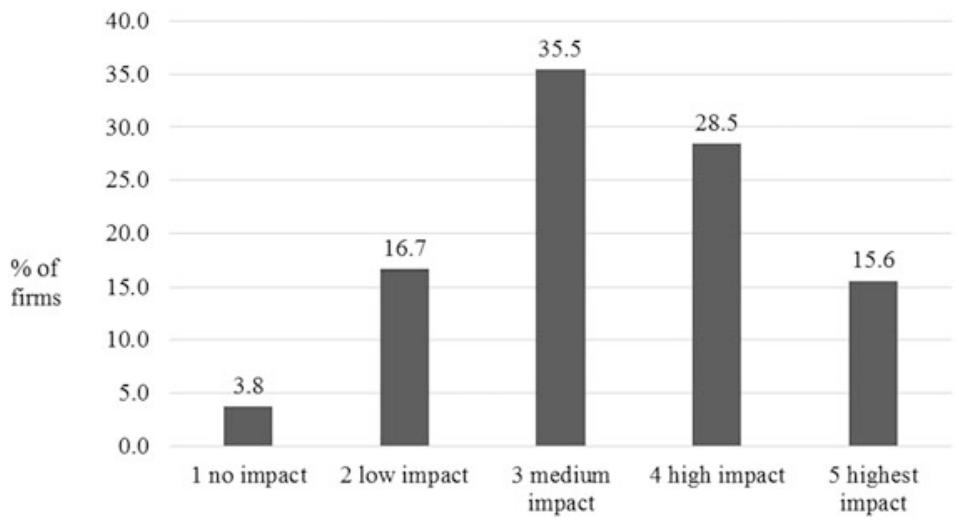

Fig. 4.1 The impact of the current economic and financial crisis. Source: "Osservatorio Impresa Monza e Brianza" (2012)

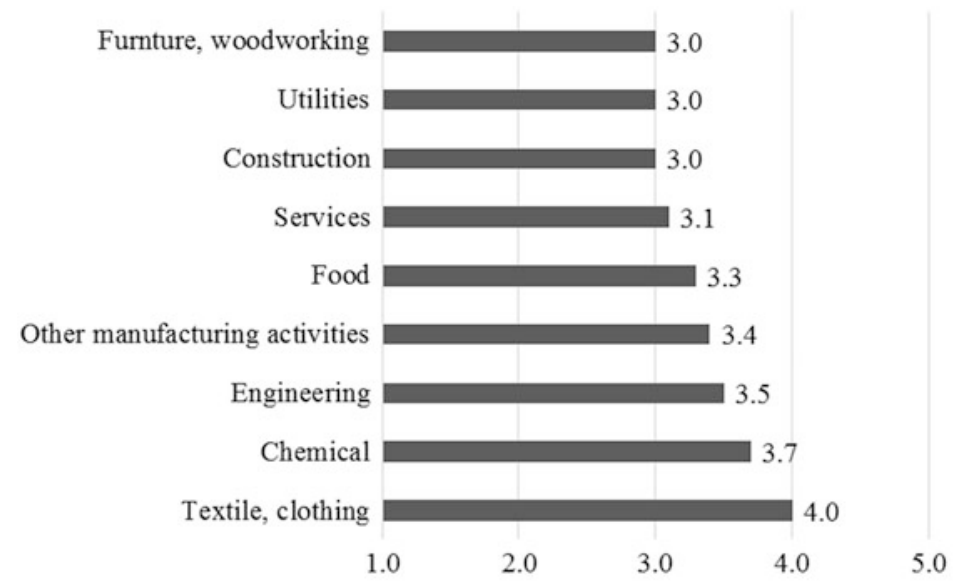

1 no impact; 2 low impact; 3 medium impact; 4 high impact; $5=$ highest impact

Fig. 4.2 The impact of the current economic and financial crisis on individual sectors. Source: "Osservatorio Impresa Monza e Brianza" (2012) 


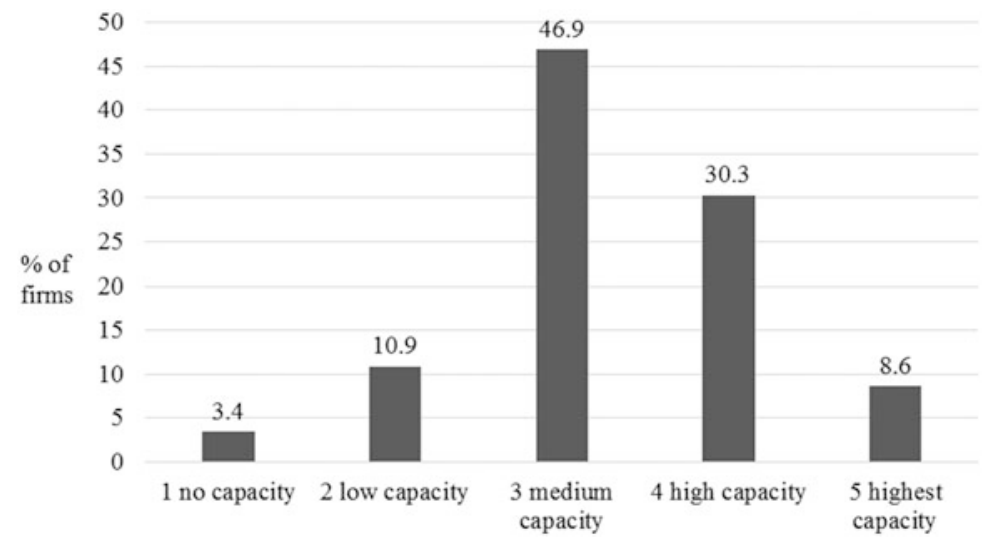

Fig. 4.3 The capacity of respondents to get out of the crisis. Source: "Osservatorio Impresa Monza e Brianza" (2012)

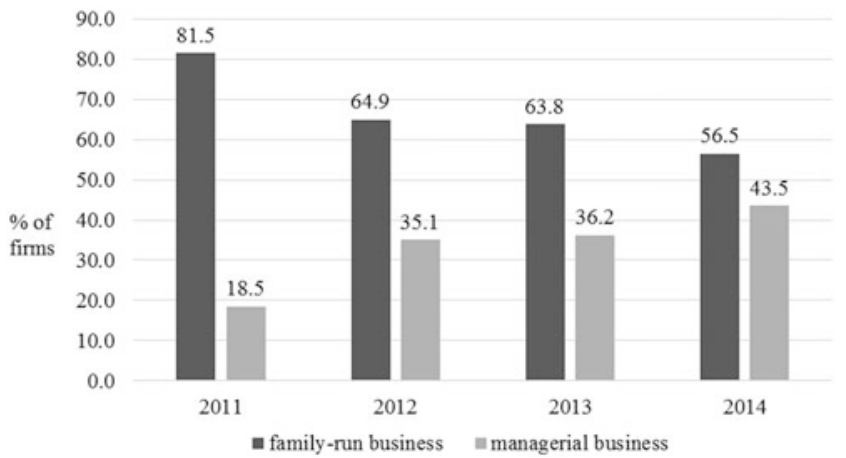

Fig. 4.28 Family businesses in the four editions of the survey. Source: "Osservatorio Impresa Monza e Brianza" (2011, 2012, 2013, 2014) 